\title{
Extremal Paths on a Random Cayley Tree
}

\author{
Satya N. Majumdar ${ }^{1,2}$ and P. L. Krapivsky ${ }^{1,3}$ \\ ${ }^{1}$ Laboratoire de Physique Quantique (UMR C5626 du CNRS), Université Paul Sabatier, 31062 Toulouse Cedex, France \\ ${ }^{2}$ Tata Institute of Fundamental Research, Homi Bhabha Road, Mumbai-400005, India \\ ${ }^{3}$ Center for Polymer Studies and Department of Physics, Boston University, Boston, MA 02215, USA
}

\begin{abstract}
We investigate the statistics of extremal path(s) (both the shortest and the longest) from the root to the bottom of a Cayley tree. The lengths of the edges are assumed to be independent identically distributed random variables drawn from a distribution $\rho(l)$. Besides, the number of branches from any node is also random. Exact results are derived for arbitrary distribution $\rho(l)$. In particular, for the binary $\{0,1\}$ distribution $\rho(l)=p \delta_{l, 1}+(1-p) \delta_{l, 0}$, we show that as $p$ increases, the minimal length undergoes an unbinding transition from a 'localized' phase to a 'moving' phase at the critical value, $p=p_{c}=1-b^{-1}$, where $b$ is the average branch number of the tree. As the height $n$ of the tree increases, the minimal length saturates to a finite constant in the localized phase $\left(p<p_{c}\right)$, but increases linearly as $v_{\min }(p) n$ in the moving phase $\left(p>p_{c}\right)$ where the velocity $v_{\min }(p)$ is determined via a front selection mechanism. At $p=p_{c}$, the minimal length grows with $n$ in an extremely slow double logarithmic fashion. The length of the maximal path, on the other hand, increases linearly as $v_{\max }(p) n$ for all $p$. The maximal and minimal velocities satisfy a general duality relation, $v_{\min }(p)+v_{\max }(1-p)=1$, which is also valid for directed paths on finite-dimensional lattices.
\end{abstract}

PACS numbers: 05.40.-a, 64.60.Cn, 02.50.-r

\section{INTRODUCTION}

Optimization lies at the heart of a vast number of phenomena: Particles "seek" paths with minimal action, species "try" to maximize fitness, companies minimize cost. Many optimization problems, e.g., the traveling salesman problem, are notoriously hard [1]. Additionally, optimization problems often involve randomness which makes them even more complicated. When the number of random entries is very large, however, optimization problems might become simpler thanks to the "concentration of the measure". This means that the probability distribution of some random variable becomes highly localized, almost like a delta function concentrated around its average value. This phenomenon is well-known in probability theory where, for instance, it accounts for the fact that after flipping a coin $N$ times, the probability that the number of heads exceeds $N / 2$ by more than, say, $100 \sqrt{N}$ is about $10^{-800}$. Below, we shall investigate an optimization problem in random media which demonstrates this concentration of the measure in the strongest sense (the variance of the optimized quantity is finite) and displays an unbinding phase transition. A remarkable hidden connection with traveling wave phenomena allows to explain both the concentration of the measure and the phase transition.

The optimization problem considered in this work can be formulated as follows: Take a rooted tree whose bonds have random lengths and find descending paths of extremal total length. We assume that a random length (or energy) is assigned to each bond of the tree, and that lengths are independent and chosen from the same probability distribution $\rho(l)$. The total length of a path is the sum of lengths of the bonds along the path, and we want to determine the minimal (maximal) length among the paths from the root to the bottom of the tree. We focus on a rooted Cayley tree where each node, except the root, has coordination number 3 ; the coordination number of the root is 2. However, our main results can be easily generalized to the case of a tree with arbitrary coordination number, and even to trees where the coordination number at any node is random.

Similar problems arise in numerous fields ranging from computer science [2] to condensed matter physics [3, 4], where it is known as the problem of directed polymers in random media. Indeed, the minimal path problem can be considered as the zero temperature limit of the problem of directed polymer on Cayley trees. Directed polymers on Cayley trees have been investigated in the past [5], 6], and recently this problem has resurfaced in a surprisingly large number of apparently unrelated problems [7 9. However, the emphasis of Refs. [5] 9] was on the spin glass like transition occurring at a finite temperature. Here, we consider exactly zero temperature and focus on the unbinding transition driven by the parameter $p$ of the bimodal distribution, $\rho(l)=p \delta_{l, 1}+(1-p) \delta_{l, 0}$. Recently, similar unbinding (or "depinning") phase transitions have been found in a number of non-equilibrium processes without quenched disorder [11 14].

Let us first outline our main results. We derive exactly the statistics of both the minimal and maximal path on a random Cayley tree for arbitrary distribution $\rho(l)$ of the edge lengths. We find a class of distributions for which the minimal path undergoes an unbinding phase transition from a localized phase to a moving phase as a parameter of the distribution is varied. A particular example, which we study in detail in this paper, is the bimodal distribution. In the bimodal case, length of each 
bond can be either 1 with probability $p$ or 0 with probability $(1-p)$. For any path starting at the root and moving down, the length of the path is the sum of the lengths of the bonds along the path, i.e., the number of 1 's along the path. There are $2^{n}$ paths from the root to the $n^{\text {th }}$ level. We show that the minimal length exhibits an unbinding transition as the parameter $p$ varies between 0 and 1 . There exists a critical value $p_{c}=1 / 2$ such that the average minimal length grows linearly with $n$ when $p>1 / 2$, but gets pinned, i.e., remains finite for large $n$ when $p<1 / 2$. More specifically, we find that for large $n$, the average minimal length $\left\langle L_{n}^{\min }\right\rangle$ behaves as

$$
\left\langle L_{n}^{\min }\right\rangle \simeq \begin{cases}v_{\min }(p) n & p>1 / 2 \\ (\ln 2)^{-1} \ln \ln n & p=1 / 2 \\ \text { finite } & p<1 / 2\end{cases}
$$

For $p>1 / 2$, we shall later compute the minimal "velocity" $v_{\min }(p)$ exactly. The average maximal length, on the other hand, behaves as

$$
\left\langle L_{n}^{\max }\right\rangle \simeq v_{\max }(p) n \quad \text { for all } \mathrm{p}
$$

where $v_{\max }(p)+v_{\min }(1-p)=1$. This duality relation is very general and valid for directed paths on arbitrary graphs, i.e., for finite-dimensional lattices. We also derive a generalized duality relation between minimal and maximal velocities for a certain class of bounded distributions.

The minimal length problem on a rooted Cayley tree, where the coordination number at each node is random, also displays an unbinding transition at $p_{c}=1-b^{-1}$, where $b$ is the average number of branches per node. At the critical point, the average minimal length $\left\langle L_{n}^{\min }\right\rangle$ exhibits the same double logarithmic growth for any $b>1$, and above $p_{c}$ the minimal length grows linearly with $n$ with velocity $v_{\min }(p, b)$.

The rest of this paper is organized as follows. In Section II, we study the unbinding transition of the minimal path for the special bimodal distribution in detail. In Section III, we study the maximal path for the bimodal case and derive the duality relation. In Section IV, we generalize the results to a Cayley tree with random branching. In Section IV, we go beyond the bimodal distribution and obtain generalized results for arbitrary distribution of the edge length. Finally we conclude in Section VI with a brief summary and outlook.

\section{MINIMAL PATH(S)}

Instead of studying $P_{n}^{\min }(x)=\operatorname{Prob}\left(L_{n}^{\min }=x\right)$, it proves convenient to consider the cumulative distribution, $P_{n}(x)=\operatorname{Prob}\left(L_{n}^{\min } \geq x\right)$. Clearly, $P_{n}(x)$ is the probability that all possible paths to the $n^{\text {th }}$ level have lengths $\geq x$. Once we get $P_{n}(x)$, the distribution of the minimal length is found from the obvious identity $P_{n}^{\min }(x)=P_{n}(x)-P_{n}(x+1)$.

It is easy to see that $P_{n}(x)$ satisfies the following recursion relation

$$
P_{n+1}(x)=\left[(1-p) P_{n}(x)+p P_{n}(x-1)\right]^{2} .
$$

This relation can be derived by analyzing various possibilities for the lengths of the two edges issuing from the root and taking into account that two subsequent daughter trees are statistically independent. For instance, both lengths are equal to zero with probability $(1-p)^{2}$, and paths in the subsequent trees have lengths $\geq x$ with probability $P_{n}^{2}(x)$; this provides the contribution $(1-p)^{2} P_{n}^{2}(x)$ to $P_{n+1}(x)$. Similarly, one gets the contributions $2 p(1-p) P_{n}(x-1) P_{n}(x)$ and $p^{2} P_{n}^{2}(x-1)$, which sum up to Eq. (3).

We must solve Eq. (3) subject to the initial condition

$$
P_{0}(x)= \begin{cases}1 & x \leq 0 \\ 0 & x>0\end{cases}
$$

Clearly, the minimal length $L_{n}^{\min }$ is a random variable which takes values between 0 and $n$, and Eqs. (3)-(4) indeed show that $P_{n}(x)=1$ for $x \leq 0$ and $P_{n}(x)=0$ for $x>n$.

When $n$ grows, $L_{n}^{\min }$ increases as well and thus it should reach a limit. For $p<1 / 2$, this limit is finite, i.e., one can find an infinite path with only finite number of edges of length 1. Mathematically, it means that $P_{n}(x)$ approaches a stationary distribution, $P_{n}(x) \rightarrow P(x)$, which satisfies

$$
P(x)=[(1-p) P(x)+p P(x-1)]^{2} .
$$

Starting from $P(0)=1$, one computes $P(x)$ recursively: $P(1)=p^{2}(1-p)^{-2}$, etc. $P(x)$ vanishes extremely fast in the large $x$ limit: $\ln P(x) \sim 2^{x} \ln p$.

For $p \geq 1 / 2$, the minimal length grows as $n$ increases. To understand why this is so, recall that $P_{n}(0) \equiv 1$ and look at $P_{n}(1)$. By inserting $P_{n}(1)=1-q_{n}$ into Eq. (3) we find $q_{n+1}=2(1-p) q_{n}-(1-p)^{2} q_{n}^{2}$. Hence $P_{n}(1) \rightarrow 1$ for $p \geq 1 / 2$. Proceeding this line of reasoning one can verify that $P_{n}(x) \rightarrow 1$ when $n \rightarrow \infty$. Additionally, we see the difference between the cases of $p>1 / 2$ and $p=1 / 2$ : In the former situation, $P_{n}(x) \rightarrow 1$ exponentially fast, while in the latter situation the approach is algebraic. Therefore, the size of the region where $P_{n}(x)$ is close to 1 should grow linearly with $n$ when $p>1 / 2$. Thus the distribution $P_{n}(x)$ approaches the traveling wave form (see Fig. (1)),

$$
P_{n}(x) \rightarrow \mathcal{P}^{2}(y), \quad y=x-\left\langle L_{n}^{\min }\right\rangle .
$$

In Eq. (6), we have chosen $y=x-\left\langle L_{n}^{\min }\right\rangle$ as the wave variable. One can justify this choice by noting that the relation $P_{n}^{\min }(x)=P_{n}(x)-P_{n}(x+1)$ leads to identity

$$
\left\langle L_{n}^{\min }\right\rangle=\sum_{x=0}^{\infty} x\left[P_{n}(x)-P_{n}(x+1)\right]=\sum_{x=1}^{\infty} P_{n}(x),
$$


and hence $\left\langle L_{n}^{\min }\right\rangle$ is indeed an appropriate characterization of the location of the front. By inserting the wave form (6) into Eq. (3) and using $\left\langle L_{n}^{\mathrm{min}}\right\rangle \simeq v n$ we find,

$$
\mathcal{P}(y-v)=(1-p) \mathcal{P}^{2}(y)+p \mathcal{P}^{2}(y-1),
$$

The solution of this equation looks like a $[1-0]$ waveform with $P(y) \rightarrow 1$ as $y \rightarrow-\infty$ and $P(y) \rightarrow 0$ as $y \rightarrow \infty$. While it is very hard to solve this nonlinear, nonlocal equation exactly, the velocity can be found by analyzing the tail region, $y \rightarrow-\infty$, where $1-\mathcal{P}(y)$ is small. Linearizing Eq. (8) in this region and noting that it admits an exponential solution, $1-\mathcal{P}(y) \sim e^{\lambda y}$, one finds that the velocity $v_{\lambda}$ is related to the decay exponent $\lambda$ via

$$
v_{\lambda}=-\frac{\ln \left[2(1-p)+2 p e^{-\lambda}\right]}{\lambda} .
$$

When $\lambda>\ln \frac{2 p}{2 p-1}$, the velocity $v_{\lambda}$ is positive. While any such $\lambda$ is in principle allowed, and thus the entire velocity range of is feasible, a particular value is actually selected. This is similar to the velocity selection in a large class of problems with a traveling wave front [15, 16]. It is well known that for a wide class of initial conditions, the extremum value is chosen. From this general front selection principle one can infer that in the present case the selected value $\lambda=\lambda^{*}(p)$ corresponds to the maximum of $v_{\lambda}$, and hence the selected "velocity" is $v_{\min }(p)=v_{\lambda^{*}}$. Thus, $\lambda^{*}$ is a solution of the equation

$$
\ln \left[2(1-p)+2 p e^{-\lambda^{*}}\right]+\frac{p \lambda^{*} e^{-\lambda^{*}}}{1-p+p e^{-\lambda^{*}}}=0,
$$

and the selected velocity is

$$
v_{\min }(p)=\frac{p e^{-\lambda^{*}}}{1-p+p e^{-\lambda^{*}}} .
$$

Although it seems impossible to provide an explicit expression for $v_{\min }(p)$, one can easily probe its behavior in the limiting cases $p \downarrow \frac{1}{2}$ and $p \uparrow 1$. In these respective limits, one gets

$$
v_{\min }(p) \simeq \frac{2 p-1}{\ln \frac{1}{2 p-1}}
$$

and

$$
1-v_{\min }(p) \simeq \frac{\ln 2}{\ln \frac{1}{1-p}} .
$$

One can also derive the leading correction to the dominant linear behavior of $\left\langle L_{n}^{\mathrm{min}}\right\rangle$,

$$
\left\langle L_{n}^{\min }\right\rangle \simeq v_{\min }(p) n+\frac{3}{2 \lambda^{*}} \ln n .
$$

The logarithmic correction becomes especially important when $p \downarrow \frac{1}{2}$, as can be seen from the asymptotics

$$
\left\langle L_{n}^{\min }\right\rangle \simeq \frac{\epsilon n}{\ln (1 / \epsilon)}+\frac{3 \ln n}{2 \ln (1 / \epsilon)}, \quad \epsilon=2 p-1 .
$$

The logarithmic correction to the front position was first derived by Bramson in the context of a reactiondiffusion equation 17, 15, and was subsequently rederived and generalized by a number of authors 1820 . Our derivation of Eq. (14) follows an approach of Ref. [18], and we only outline the main steps. First, we need to examine the exact equation (3) rather than the $n \rightarrow \infty$ limit, Eq. (8), which was sufficient to determine the velocity. However, the analysis is still performed in the region far behind the front, so we can use the linearized version of Eq. (3). Writing $P_{n}(x)=1-Q_{n}(x)$, plugging it into Eq. (3), and ignoring quadratic terms gives

$$
Q_{n+1}(x)=2(1-p) Q_{n}(x)+2 p Q_{n}(x-1) .
$$

Now we seek a solution which has the form

$$
Q_{n}(x)=n^{\alpha} G\left(y n^{-\alpha}\right) e^{\lambda^{*} y},
$$

with $y=x-\left\langle L_{n}^{\min }\right\rangle$ as previously and yet undetermined exponent $\alpha$ and the scaling function $G(z)$. The correction to the leading term in the front position should be incorporated as,

$$
\left\langle L_{n}^{\min }\right\rangle \simeq v_{\min }(p) n+c(n)
$$

where the function $c(n)$ is yet undetermined. Plugging Eqs. (17)-(18) into Eq. (16) we find that different leading orders are compatible provided that $\alpha=1 / 2$ and $c(n)=\beta \ln n$ with some constant $\beta$. Additionally, the scaling function $G(z)$ satisfies a parabolic cylinder equation

$$
\frac{d^{2} G}{d z^{2}}+z \frac{d G}{d z}+\left(2 \beta \lambda^{*}-1\right) G(z)=0 .
$$

Equation (19) should be solved subject to the boundary conditions $G(z) \rightarrow 0$ as $z \rightarrow \infty$ as $Q_{n}(x)$ must vanish for $x \rightarrow \infty$ and $G(z) \sim z$ for $z \rightarrow 0$ to ensure that $Q_{n}(x)$ is independent on $n$ in the limit $n \rightarrow \infty$. The boundary condition in the large $z$ limit selects one of the two possible solutions: $G(z)=A \exp \left(-z^{2} / 4\right) D_{2\left(\beta \lambda^{*}-1\right)}(z)$, where $D_{\nu}$ is the parabolic cylinder function with index $\nu$. The second boundary condition $G(z) \sim z$ fixes the index of the parabolic cylinder function, $2\left(\beta \lambda^{*}-1\right)=1$. Hence, $\beta=3 / 2 \lambda^{*}$ thus completing the derivation of Eq. (14). Note that the sign of the logarithmic correction term in Eq. (14) is positive. This is different from the most earlier studied problems 18 20 although the positive sign has been occasionally seen, see Ref. [21].

In the the critical case of $p=1 / 2$, the convergence of the distribution $P_{n}(x)$ towards the asymptotic value is only algebraic, and therefore the front propagates extremely slowly. The simplest way to determine the rate of propagation is again to look at $P_{n}(x)$ far behind the 
front. Writing $P_{n}(x)=1-Q_{n}(x)$ and plugging it into Eq. (3) yields

$$
\begin{aligned}
Q_{n+1}(x) & =Q_{n}(x)+Q_{n}(x-1) \\
& -\left[\frac{Q_{n}(x)+Q_{n}(x-1)}{2}\right]^{2} .
\end{aligned}
$$

$Q_{n}(x)=0$ for $x \leq 0$, so the first non-trivial $Q_{n}$ 's are $Q_{n}(1) \equiv q(n)$. From Eq. (20), $q(n+1)=q(n)-\frac{q^{2}(n)}{4}$. In the large $n$ limit, we can employ the continuum approximation to get $\frac{d q}{d n}=-\frac{q^{2}}{4}$ and thence $Q_{n}(1) \simeq 4 n^{-1}$. Similarly, we find the asymptotics $Q_{n}(2) \simeq 4 n^{-1 / 2}$. Generally, Eq. (20) implies $Q_{n}(x) \simeq 2 \sqrt{Q_{n}(x-1)}$, from which

$$
Q_{n}(x) \simeq 4 n^{-2^{-(x-1)}}=4 \exp \left(-2 e^{\ln \ln n-x \ln 2}\right) .
$$

This demonstrates that at the critical point, $Q_{n}(x)$ also has a traveling wave form, $Q_{n}(x)=g\left(x-x_{n}\right)$, with the front at $x_{n} \simeq(\ln 2)^{-1} \ln \ln n$.

Equation (21) formally applies for $x \ll x_{n}$. To investigate $Q_{n}(x)$ in the entire range of $x$, we again use the fact that the distribution $Q_{n}(x)$ should approach a traveling wave form, $Q_{n}(x) \rightarrow Q(y)$ with $y=n^{-2^{-(x-1)}}$, as $n \rightarrow \infty$. By inserting this into (20) and taking $n \rightarrow \infty$ limit, we arrive at

$$
Q(y)=2 \sqrt{Q\left(y^{2}\right)}-Q\left(y^{2}\right) .
$$

From this equation, one finds

$$
Q(y)=4 y-4 y^{2}-2 y^{3}+\ldots \quad \text { for } \quad y \downarrow 0 .
$$

The first term in this expansion indeed agrees with Eq. (21). Similarly, one gets

$$
1-Q(y) \sim \exp \left[-\frac{\text { const }}{1-y}\right] \text { for } y \uparrow 1 .
$$

To provide a more rigorous derivation of the growth law for $\left\langle L_{n}^{\mathrm{min}}\right\rangle$, note that $\left\langle L_{n}^{\mathrm{min}}\right\rangle=\sum_{x>1} P_{n}(x)=n-$ $\sum_{x \geq 1} Q_{n}(x)$. Inserting $Q_{n}(x)=Q(y)$ and replacing the sum by an integral (valid for large $n$ ), we get,

$$
\left\langle L_{n}^{\min }\right\rangle \approx n-\frac{1}{\ln 2} \int_{2^{n-1} \ln n}^{\ln n} Q\left(e^{-z}\right) \frac{d z}{z} .
$$

In the limit of large $n$, the most important contribution in the integral comes from its lower limit. Using $Q(y) \rightarrow 1$ as $y \rightarrow 1$ (see Eq. (24)), we arrive at the desired result,

$$
\left\langle L_{n}^{\min }\right\rangle \simeq(\ln 2)^{-1} \ln \ln n .
$$

\section{MAXIMAL PATH(S)}

We now turn to the statistics of $L_{n}^{\max }$, the length of the maximal path for the bimodal distribution. It is now convenient to define $R_{n}(x)=\operatorname{Prob}\left(L_{n}^{\max } \leq x\right)$. By proceeding as in the minimal length problem, it is easy to show that $R_{n}(x)$ evolves according to the same equation (3) with $P$ replaced by $R$, i.e.,

$$
R_{n+1}(x)=\left[(1-p) R_{n}(x)+p R_{n}(x-1)\right]^{2} .
$$

The only difference from the minimal case is in the initial condition. Instead of Eq. (4), we now have

$$
R_{0}(x)= \begin{cases}1 & x \geq 0 \\ 0 & x<0\end{cases}
$$

This difference in the initial condition, however, leads to a different behavior of the maximal front as demonstrated below.

Not surprisingly, equation (27) admits a traveling wave solution, $R_{n}(x)=R\left(y=x-v_{\max } n\right)$ for all $p$ (see Fig. (2)). However, in contrast to the $[1-0]$ waveform of the minimal case, the function $R(y)$ now looks like a $[0-1]$ waveform with $R(y) \rightarrow 0$ as a double exponential behind the front $(y \rightarrow-\infty)$ and $R(y) \rightarrow 1$ exponentially ahead the front $(y \rightarrow \infty)$. We then analyze the wavefront near the 'forward' tail, as opposed to the 'backward' tail of the minimal problem. Substituting $1-R(y) \sim e^{-\mu y}$, we find that there exists a family of traveling wave solutions with velocity $v_{\mu}$ parametrized by $\mu$,

$$
v_{\mu}=\frac{\ln \left[2(1-p)+2 p e^{\mu}\right]}{\mu} .
$$

This velocity $v_{\mu}$ has a unique minimum at $\mu=\mu^{*}$. By the front selection mechanism, this minimum value $v_{\mu^{*}}$ is selected as the velocity $v_{\max }(p)$ of the front. Thus, we have

$$
\ln \left[2(1-p)+2 p e^{\mu^{*}}\right]-\frac{p \mu^{*} e^{\mu^{*}}}{1-p+p e^{\mu^{*}}}=0,
$$

and the selected maximal velocity is

$$
v_{\max }(p)=\frac{p e^{\mu^{*}}}{1-p+p e^{\mu^{*}}} .
$$

Using these results together with Eqs. (10) and (11), one immediately finds

$$
v_{\min }(p)+v_{\max }(1-p)=1 .
$$

This is the duality relation. Unlike the behavior of the minimal length, the maximal length does not undergo an unbinding transition in the sense that it increases linearly with $n$ for large $n$ for all $0 \leq p \leq 1$. However, there is still a transition at $p=1 / 2$ : The velocity $v_{\max }(p)$ increases from 0 to 1 as $p$ increases from 0 to $1 / 2$ and then sticks to 1 for $p \geq 1 / 2$ (see Fig. (3)).

For the maximal front also, one can easily compute the sub-leading logarithmic correction to the front position. Proceeding as in the minimal case, we find 


$$
\left\langle L_{n}^{\max }\right\rangle \simeq v_{\max }(p) n-\frac{3}{2 \mu^{*}} \ln n .
$$

Note the negative sign in the correction term, as opposed to the positive sign for the minimal case in Eq. (14). This is also consistent with the duality relation in Eq. (32). Thus, while the coefficient $3 / 2$ of the logarithmic correction term seems to be universal not just in the present problem but in many other cases [18 20], the sign of the correction term is not and can either be positive [21] or negative 18 20].

The duality relation (32) can also be derived by a general argument which does not depend on the structure of the underlying tree and therefore is valid for extremal directed paths on arbitrary graphs, in particular, on arbitrary lattices. The argument follows from the observation that for bimodal distribution, if one replaces the 0's by 1 's and the 1's by 0's on the minimal path (and thereby replacing $p$ by $1-p$ ), then the minimal path becomes the maximal path. Let $n_{0}$ and $n_{1}$ denote respectively the number of 0's and 1's on the minimal path. Evidently, $n_{0}+n_{1}=n$. Then clearly, $L_{n}^{\min }(p)=n_{1}$ and by duality, $L_{n}^{\max }(1-p)=n_{0}$. Adding the two quantities, we get $L_{n}^{\min }(p)+L_{n}^{\max }(1-p)=n$. Dividing by $n$, we immediately get the duality relation Eq. (32). Below, we shall derive a more generalized version of this duality relation which goes beyond the bimodal distribution.

\section{GENERALIZATION TO TREES WITH RANDOM BRANCHING}

The generalization of the above analysis to the case of the rooted Cayley tree with a random number of branches is straightforward. Let $b_{m}$ is the probability that the number of branches is equal to $m$ and $b$ is the average number of branches, $b=\sum_{m \geq 1} m b_{m}$. Equation (3) is replaced by

$$
P_{n+1}(x)=\sum_{m=1}^{\infty} b_{m}\left[(1-p) P_{n}(x)+p P_{n}(x-1)\right]^{m},
$$

and the subsequent analysis repeats the steps detailed in the case of the binary tree. In particular, $P_{n}(x)$ approaches the stationary distribution for $p<p_{c}$, while above $p_{c}$ the minimal length grows according to Eq. (14) with $\lambda^{*}$ a solution to equation

$$
\ln \left[b(1-p)+b p e^{-\lambda^{*}}\right]+\frac{p \lambda^{*} e^{-\lambda^{*}}}{1-p+p e^{-\lambda^{*}}}=1,
$$

and $v_{\min }(p)$ given by the same relation (111). At the critical point $p_{c}=1-b^{-1}$, the minimal length is given by the same expression (26) for any tree. Also, the velocity of the maximal path can be determined from the general duality relation in Eq. (32).

\section{GENERALIZATION TO ARBITRARY DISTRIBUTIONS}

We now consider the statistics of extreme path(s) on a binary tree for arbitrary distribution $\rho(l)$ of the edge lengths. Let us first consider the minimal path(s). The velocity of the minimal path for an arbitrary distribution can be extracted from earlier results by Derrida and Spohn [5] which we re-derive below for the sake of completeness. We consider again $P_{n}(x)=\operatorname{Prob}\left(L_{n}^{\min } \geq x\right)$. Proceeding as in the case of the bimodal distribution, one finds that the appropriate generalization of Eq. (3) is given by

$$
P_{n+1}(x)=\left[\int_{0}^{\infty} d l \rho(l) P_{n}(x-l)\right]^{2} .
$$

with the same initial condition, Eq. (4), as earlier. The distribution of the minimal length is then given by $P_{n}^{\min }(x)=-d P_{n}(x) / d x$ and the average minimal length is $\left\langle L_{n}^{\min }\right\rangle=\int_{0}^{\infty} P_{n}(x) d x$.

Defining $P_{n}(x)=G_{n}^{2}(x)$, we recast Eq. (36) into

$$
G_{n+1}(x)=\int_{0}^{\infty} d l \rho(l) G_{n}^{2}(x-l) .
$$

For any given $n, G_{n}(x) \rightarrow 1$ as $x \rightarrow-\infty$ and $G_{n}(x) \rightarrow 0$ as $x \rightarrow \infty$. Substituting $G_{n}(x)=1-g_{n}(x)$ in Eq. (37) and using normalization of $\rho(l)$ one finds,

$$
g_{n+1}(x)=\int_{-\infty}^{x} d y \rho(x-y)\left[2 g_{n}(y)-g_{n}^{2}(y)\right]
$$

where $g_{n}(x) \rightarrow 0$ as $x \rightarrow-\infty$ and $g_{n}(x) \rightarrow 1$ as $x \rightarrow+\infty$. We analyze the above equation in the 'backward' tail region, i.e., when $x \rightarrow-\infty$. In this limit, one can neglect the nonlinear term in $g_{n}$ inside the integral in Eq. (38). The resulting linear equation admits a traveling wave solution of the form, $g_{n}(x)=e^{\lambda\left(x-v_{\lambda} n\right)}$, with

$$
v_{\lambda}=-\frac{1}{\lambda} \ln \left[2 \int_{0}^{\infty} d z e^{-\lambda z} \rho(z)\right] .
$$

For generic length distributions $\rho(l)$, this function has a maximum at $\lambda=\lambda^{*}$ and by the general velocity selection principle, this maximum velocity is selected, i.e., $v_{\min }=v_{\lambda^{*}}$.

The question we are interested in is for which class of distributions $\rho(l)$, an unbinding transition can occur for the minimal path. Such a transition will occur if the velocity $v_{\lambda}$ in Eq. (39) ceases to have a unique maximum. By analyzing Eq. (39) one sees that this can happen only if $\rho(l)$ has a nonzero delta-function weight at $l=0$, i.e., when $\rho(l)=a \delta(l)+(1-a) f(l)$ with $0<a<1$ and $f(l)$ is some positive function with $\int_{0}^{\infty} d l f(l)=1$. The unbinding transition occurs as the parameter $a$ is varied. Note that the positivity condition of velocity in Eq. (39) 
demands that $2 a<1$. Thus the critical point always occurs at $a=1 / 2$. The bimodal distribution considered in the previous sections is a special case of this class of distributions with $a=1-p$ and $f(l)=\delta(l-1)$.

For $a<1 / 2$, the average minimal length increases linearly with $n$ for large $n$ with the velocity $v_{\lambda^{*}}$ obtained from Eq. (39). For $a>1 / 2$, the function $P_{n}(x)$ reaches a stationary distribution for large $n$ and hence the $\left\langle L_{n}^{\min }\right\rangle$ saturates to a nonzero constant as $n \rightarrow \infty$. What happens at the critical point $a=1 / 2$ for generic distributions $f(l)$ 's? For $a=1 / 2$, the Eq. (37) reduces to,

$$
G_{n+1}(x)=\frac{1}{2} G_{n}^{2}(x)+\frac{1}{2} \int_{0}^{\infty} d l f(l) G_{n}^{2}(x-l) .
$$

Detailed analysis of the above equation reveals a rather remarkable universal property at the critical point. It turns out that for generic $f(l)$, there are only two possible behaviors of $\left\langle L_{n}^{\min }\right\rangle=\int_{0}^{\infty} d x G_{n}^{2}(x)$, depending on whether the function $f(l)$ has a gap or not near $l=0$. If $f(l)$ does not have a gap at $l=0$, it turns out that as $n \rightarrow \infty, G_{n}(x)$ in Eq. (40) tends to a stationary distribution and hence $\left\langle L_{n}^{\min }\right\rangle \rightarrow$ const. In the opposite case, when $f(l)$ has a gap $\Delta$ near $l=0$, it turns out that as $n \rightarrow \infty$,

$$
\left\langle L_{n}^{\min }\right\rangle \simeq \frac{\Delta}{\ln 2} \ln \ln n .
$$

The bimodal distribution corresponds to the special case $\Delta=1$.

These general results are best demonstrated by specific examples which can be worked out explicitly. Let us first consider an example which is gapless, such as the exponential distribution, $f(l)=e^{-l}$. Remarkably, Eq. (40) can be solved exactly in this case. It is easy to verify that Eq. (40) admits an exponential solution, $G_{n}(x)=A_{n} e^{-x}$, where $A_{n+1}=\left(1+A_{n}^{2}\right) / 2$ with $A_{0}=0$. As $n \rightarrow \infty, A_{n}$ approaches to 1 (more precisely, $A_{n} \simeq 1-2 / n$ ). Therefore, $\left\langle L_{n}^{\min }\right\rangle=\int d x G_{n}^{2}(x)=A_{n}^{2} / 2$ approaches to $1 / 2$ as $n \rightarrow \infty$. Next we consider a distribution with gap such as $f(l)=\frac{1}{4} \delta(l-1)+\frac{1}{4} \delta(l-2)$. This case also can be worked out explicitly following the same steps as used for the bimodal case. It turns out that essentially all the steps are identical to the bimodal case, except that $n$ in Eq. (26) gets replaced by $n / 2$. But this does not change the leading asymptotic behavior for large $n$ which is still given by Eq. (26).

We now turn to the maximal path(s) for an arbitrary distribution $\rho(l)$. As in the case of the bimodal distribution, $R_{n}(x)=\operatorname{Prob}\left(L_{n}^{\max } \leq x\right)$ satisfies the same equation (36) as $P_{n}(x)$, i.e.,

$$
R_{n+1}(x)=\left[\int_{0}^{\infty} d l \rho(l) R_{n}(x-l)\right]^{2},
$$

the only difference is that the initial condition, Eq. (4), should be replaced by Eq. (28). Substituting $R_{n}(x)=$ $\left[1-s_{n}(x)\right]^{2}$, we get

$$
s_{n+1}(x)=\int_{-\infty}^{x} d y \rho(x-y)\left[2 s_{n}(y)-s_{n}^{2}(y)\right],
$$

with the boundary conditions $s_{n}(x) \rightarrow 1$ as $x \rightarrow-\infty$ and $s_{n}(x) \rightarrow 0$ as $x \rightarrow \infty$. We now have to analyze the 'forward' tail of the front, i.e., the behavior in the $x \rightarrow \infty$ limit. We first consider bounded distributions $\rho(l)$ 's with an upper cut-off $\Lambda$. In that case, the lower limit of the integration in Eq. (43) becomes $x-\Lambda$. In the tail region where $x \gg \Lambda$, one can again neglect the nonlinear term in $s_{n}$ inside the integral in Eq. (43) and the resulting linear equation admits a traveling wave solution of the form $s_{n}(x)=e^{-\mu\left(x-v_{\mu} n\right)}$, where

$$
v_{\mu}=\frac{1}{\mu} \log \left[2 \int_{0}^{\Lambda} d z e^{\mu z} \rho(z)\right] .
$$

For generic distribution, $v_{\mu}$ has a unique minimum at $\mu=\mu^{*}$ and via the front selection principle, this minimum velocity is chosen and one gets, $v_{\max }=v_{\mu^{*}}$. Comparing Eqs. (39) and (44) it becomes evident that for the bounded symmetric distributions, i.e., when $\rho(l)=$ $\rho(\Lambda-l)$, one gets the general relation,

$$
v_{\min }+v_{\max }=\Lambda .
$$

For unbounded distributions, however, one has to be careful since it is not obvious that one can neglect the nonlinear term in $s_{n}$ inside the integral in Eq. (43). Equation (44) for the maximal velocity still remains valid as long as $\rho(l)$ decays with $l$ exponentially or faster. To see this explicitly in an example, let us consider the exponential distribution, $\rho(l)=e^{-l}$. In this case, one can transform the the integral equation (43) into the following difference-differential equation,

$$
\frac{d s_{n+1}(x)}{d x}=2 s_{n}(x)-s_{n}^{2}(x)-s_{n+1}(x) .
$$

In this differential form, it is clear that for large $x$, one can neglect the nonlinear term on the right-hand side of Eq. (46). By inserting $s_{n}(x) \sim e^{-\mu\left(x-v_{\mu} n\right)}$ into resulting linear equation, one gets

$$
v_{\mu}=\frac{1}{\mu} \ln \left(\frac{2}{1-\mu}\right) .
$$

Note that the same formula is obtained if one substitutes $\rho(l)=e^{-l}$ directly into the general expression (44). Minimizing with respect to $\mu$, one gets $v_{\max }$. The corresponding minimal velocity $v_{\min }$ for the exponential distribution can be obtained from the general formula in Eq. (39) with $\rho(z)=e^{-z}$ and then maximizing with respect to $\lambda$. So, for the exponential distribution we finally get,

$$
v_{\min }=0.231961 \ldots \text { and } \quad v_{\max }=2.678346 \ldots
$$

Thus for unbounded distributions, there does not seem to exist any simple relation between minimal and maximal velocities. 


\section{CONCLUSION}

In this work, we have shown that the length of the minimal path on a Cayley tree exhibits an unbinding phase transition from a localized phase to a moving phase. This phase transition is driven by the parameter $p$ of the bimodal distribution. In the localized phase $\left(p<p_{c}\right)$, the minimal length distribution approaches a stationary depth-independent form $P^{\min }(x)$. This distribution vanishes extremely sharply, as the double exponential, in the large $x$ limit. In the moving phase $\left(p>p_{c}\right)$, the minimal length distribution $P_{n}^{\min }(x)$ approaches a traveling wave form with the front velocity relaxing algebraically, $v_{\text {min }}=v(p)+3 /\left(2 \lambda^{*} n\right)+\mathcal{O}\left(n^{-3 / 2}\right)$, in the large $n$ limit. Specifically, $P_{n}^{\min }(x)$ approaches the solitary traveling wave $\mathcal{P}(y)-\mathcal{P}(y+1)$, with $y=x-\left\langle L_{n}^{\min }\right\rangle$, the front position $\left\langle L_{n}^{\min }\right\rangle$ given by Eq. (14), and $\mathcal{P}(y)$ being a solution of Eq. (8). In the critical case, $p=p_{c}$, the minimal length distribution approaches the solitary traveling wave, although the front propagates extremely slowly, as an iterated logarithm. Given that the distribution of the minimal length is the soliton of a finite width, the variation of the minimal length is finite. In other words, $L_{n}^{\min }$ should not vary much from sample to sample.

We have also studied the complementary problem of the statistics of the maximal path. We have found that the maximal length always grows linearly with the height, with the velocity $v_{\max }$ again determined via the front selection mechanism. For the bimodal length distribution, the minimal and maximal velocities are connected via the duality relation, $v_{\min }(p)+v_{\max }(1-p)=1$, which admits extensions to finite-dimensional situations and to arbitrary bounded symmetric length distributions.

We have found that the tail of the minimal length distribution is a double exponential, in contrast with a simple exponential that occurs in most traveling wave problems [16]. This is not very surprising since similar tails were found in statistics of extremes [22]. Note, however, that our exact results exemplify extreme value statistics for correlated random variables (see 9] for a recent review), while classical results [22] correspond to the case of independent identically distributed random variables.

PLK acknowledges support from NSF, ARO, and CNRS.
[1] M. R. Carey and D. S. Johnson, Computers and Intractability: A Guide to the Theory of NP-Completeness (Freeman, New York, 1979).

[2] R. M. Karp and J. Pearl, Artificial Intelligence 21, 99 (1983); R. M. Karp and Y. Zhang, Random Structures and Algorithms, 7, 97 (1995).

[3] D. A. Huse and C. L. Henley, Phys. Rev. Lett. 54, 2708 (1985); D. A. Huse and C. L. Henley, and D. S. Fisher, Phys. Rev. Lett. 55, 2924 (1985).

[4] For a review of directed polymers in random media, see e.g. T. Halpin-Healy and Y.-C. Zhang, Phys. Reports 254, 215 (1995).

[5] B. Derrida and H. Spohn, J. Stat. Phys. 51, 817 (1988); B. Derrida, Physica Scripta T38, 6 (1991).

[6] E. Buffet, A. Patrick, and J. V. Pule, J. Phys. A 26, 1823 (1993).

[7] C. de C. Chamon, C. Mudry, and X. Wen, Phys. Rev. Lett. 77, 4194 (1996); H. E. Castillo, C. de C. Chamon, E. Fradkin, P. M. Goldbart, and C. Mudry, Phys. Rev. B 56, 10668 (1997).

[8] L. H. Tang, Phys. Rev. B 54, 3350 (1996).

[9] D. Carpentier and P. Le Doussal, cond-mat/0003281.

[10] J. Kertesz and D. E. Wolf, Phys. Rev. Lett. 62, 2571 (1989).

[11] H. Hinrichsen, R. Livi, D. Mukamel, and A. Politi, Phys. Rev. Lett. 79, 2710 (1997); Phys. Rev. E 61, R1032 (2000).

[12] T. Hwa and M. Munoz, Europhys. Lett. 41, 147 (1998).

[13] L. Giada and M. Marsili, cond-mat/0005442.

[14] S. N. Majumdar, S. Krishnamurthy, and M. Barma, Phys. Rev. E. 61, 6337 (2000).

[15] M. Bramson, Convergence of Solutions of the Kolmogorov Equation to Travelling Waves (American Mathematical Society, Providence, R.I., 1983).

[16] J. D. Murray, Mathematical Biology (Springer-Verlag, New York, 1989).

[17] M. Bramson, Commun. Pure Appl. Math. 21, 531 (1978).

[18] E. Brunet and B. Derrida, Phys. Rev. E 56, 2597 (1997).

[19] U. Ebert and B. van Saarloos, Phys. Rev. Lett. 80, 1650 (1998).

[20] For a recent review, see U. Ebert and B. van Saarloos, cond-mat/0003181.

[21] P. L. Krapivsky and S. N. Majumdar, cond-mat/000611.

[22] E. J. Gumbel, Statistics of Extremes (Columbia University Press, New York, 1958); J. Galambos, The Asymptotic Theory of Extreme Order Statistics (R. E. Krieger Publishing Co., Malabar, Florida, 1987). 


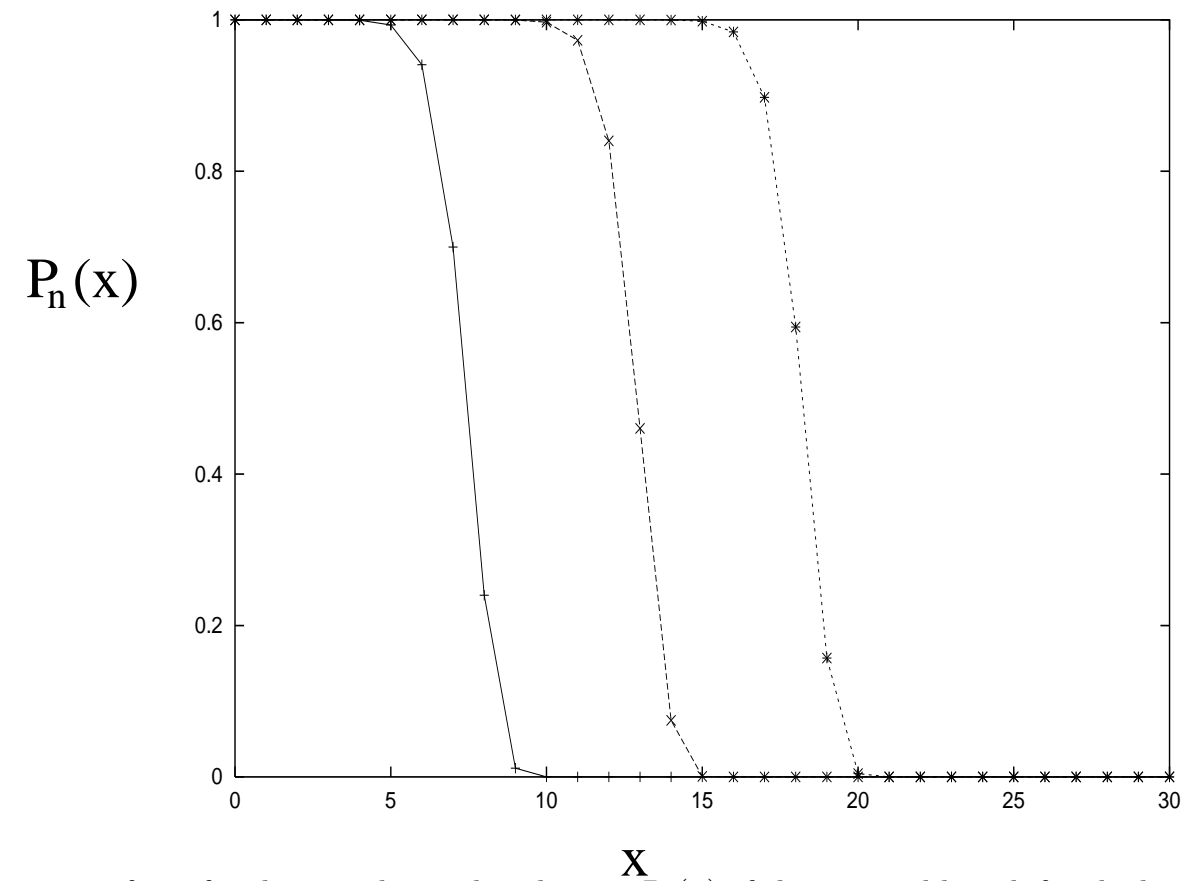

FIG. 1. The propagating front for the cumulative distribution $P_{n}(x)$ of the minimal length for the bimodal distribution with $p=0.8$.

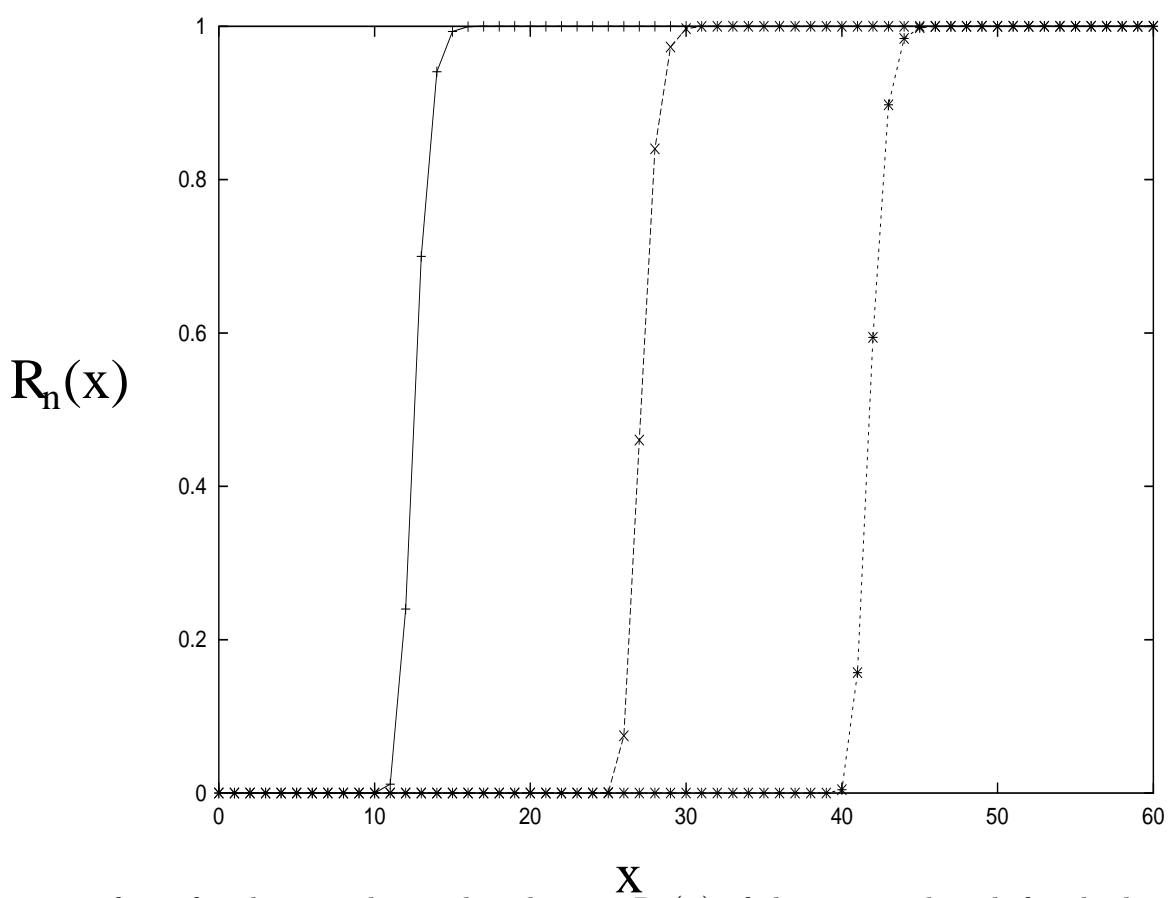

FIG. 2. The propagating front for the cumulative distribution $R_{n}(x)$ of the maximal path for the bimodal distribution with $p=0.2$. 


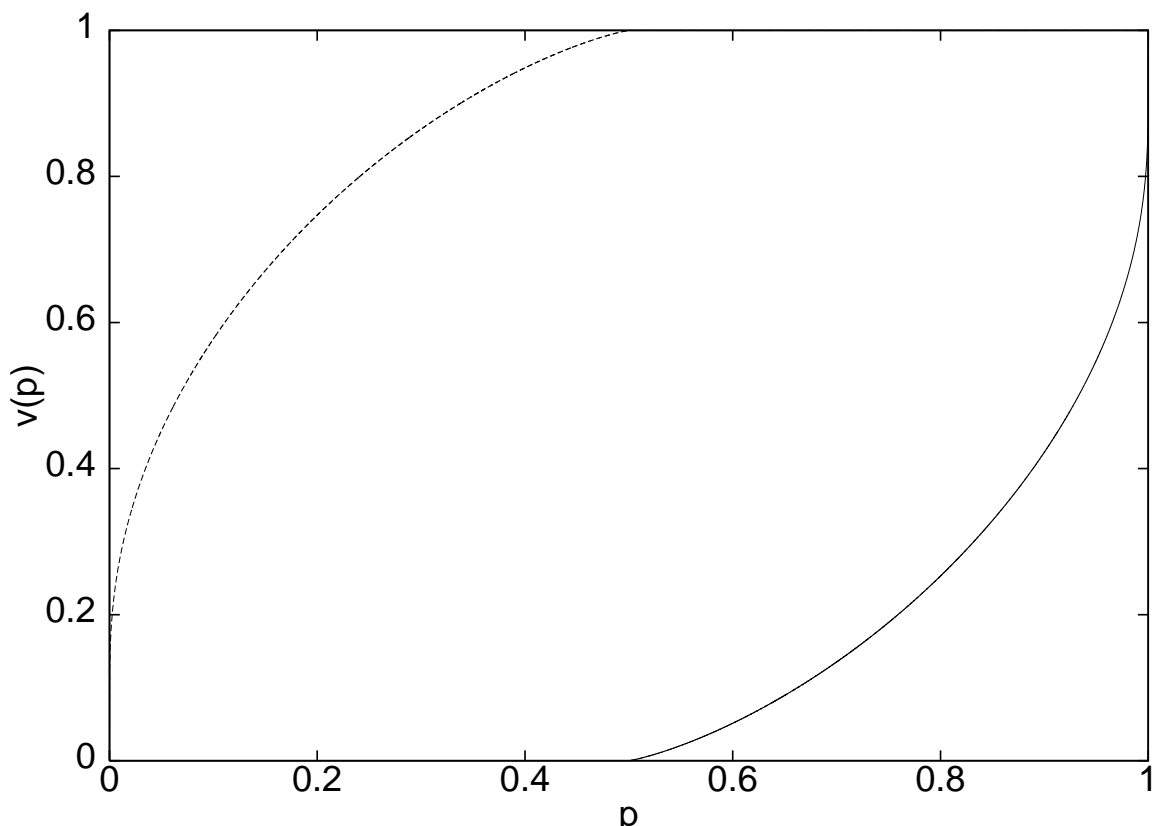

FIG. 3. The minimal and maximal velocities for the bimodal distribution as functions of $p$. The dotted line shows $v_{\text {max }}(p)$ and the solid line represents $v_{\min }(p)$. 